\title{
The Cyclic Sieving Phenomenon for Faces of Cyclic Polytopes
}

\author{
Sen-Peng $\mathrm{Eu}^{*}$ \\ Department of Applied Mathematics \\ National University of Kaohsiung, Taiwan 811, R.O.C. \\ speu@nuk.edu.tw \\ Tung-Shan $\mathrm{Fu}^{\dagger}$ \\ Mathematics Faculty \\ National Pingtung Institute of Commerce, Taiwan 900, R.O.C. \\ tsfu@npic.edu.tw \\ Yeh-Jong Pan ${ }^{\ddagger}$ \\ Department of Computer Science and Information Engineering \\ Tajen University, Taiwan 907, R.O.C. \\ yjpan@mail.tajen.edu.tw
}

Submitted: Sep 8, 2009; Accepted: Mar 17, 2010; Published: Mar 29, 2010

Mathematics Subject Classifications: 05A15, 52B15

\begin{abstract}
A cyclic polytope of dimension $d$ with $n$ vertices is a convex polytope combinatorially equivalent to the convex hull of $n$ distinct points on a moment curve in $\mathbb{R}^{d}$. In this paper, we prove the cyclic sieving phenomenon, introduced by Reiner-StantonWhite, for faces of an even-dimensional cyclic polytope, under a group action that cyclically translates the vertices. For odd-dimensional cyclic polytopes, we enumerate the faces that are invariant under an automorphism that reverses the order of the vertices and an automorphism that interchanges the two end vertices, according to the order on the curve. In particular, for $n=d+2$, we give instances of the phenomenon under the groups that cyclically translate the odd-positioned and even-positioned vertices, respectively.
\end{abstract}

\footnotetext{
*Research partially supported by the National Science Council, Taiwan under grant NSC grants 982115-M-390-002-MY3

${ }^{\dagger}$ Research partially supported by NSC grants 97-2115-M-251-001-MY2

${ }^{\ddagger}$ Research partially supported by NSC grants 98-2115-M-127-001
} 


\section{Introduction}

In [8], Reiner-Stanton-White introduced the following enumerative phenomenon for a set of combinatorial structures under an action of a cyclic group.

Let $X$ be a finite set, $X(q)$ a polynomial in $\mathbb{Z}[q]$ with the property $X(1)=|X|$, and $C$ a finite cyclic group acting on $X$. The triple $(X, X(q), C)$ is said to exhibit the cyclic sieving phenomenon (CSP) if for every $c \in C$,

$$
[X(q)]_{q=\omega}=|\{x \in X: c(x)=x\}|,
$$

where $\omega$ is a root of unity of the same multiplicative order as $c$. Such a polynomial $X(q)$ implicitly carries the information about the orbit-structure of $X$ under $C$-action. Namely, if $X(q)$ is expanded as $X(q) \equiv a_{0}+a_{1} q+\cdots+a_{n-1} q^{n-1}\left(\bmod q^{n}-1\right)$, where $n$ is the order of $C$, then $a_{k}$ counts the number of orbits whose stabilizer-order divides $k$. See [8, Theorem 7.1] for an instance of CSP on dissections of regular polygons and [1] on generalized cluster complexes.

Consider the moment curve $\gamma: \mathbb{R} \rightarrow \mathbb{R}^{d}$ defined parametrically by $\gamma(t)=\left(t, t^{2}, \ldots, t^{d}\right)$. For any $n$ real numbers $t_{1}<t_{2}<\cdots<t_{n}$, let

$$
P=\operatorname{conv}\left\{\gamma\left(t_{1}\right), \gamma\left(t_{2}\right), \ldots, \gamma\left(t_{n}\right)\right\}
$$

be the convex hull of the $n$ distinct points $\gamma\left(t_{i}\right)$ on $\gamma$. Such a polytope is called a cyclic polytope of dimension $d$. It is known that the points $\gamma\left(t_{i}\right)$ are the vertices of $P$ and the combinatorial equivalence class (with isomorphic face lattices) of polytopes with $P$ does not depend on the specific choice of the parameters $t_{i}$ (see [9]).

Let $\mathrm{CP}(n, d)$ denote a $d$-dimensional cyclic polytope with $n$ vertices. Among the $d$ dimensional polytopes with $n$ vertices, the cyclic polytope $\operatorname{CP}(n, d)$ is the one with the greatest number of $k$-faces for all $0 \leqslant k \leqslant d-1$ (by McMullen's upper bound, see [9, Theorem 8.23]). Let $f_{k}(\mathrm{CP}(n, d))$ be the number of $k$-faces of $\mathrm{CP}(n, d)$. These numbers were first determined by Motzkin [5] but no proofs were given. For a proof using the Dehn-Sommerville equations, see [3, Section 9.6]. A combinatorial proof was given by Shephard [7].

Theorem 1.1. ([7, Corollary 2]) For $1 \leqslant k \leqslant d$, the number $f_{k-1}(\mathrm{CP}(n, d))$ of $(k-1)$-faces of $\mathrm{CP}(n, d)$ is given by

$$
f_{k-1}(\mathrm{CP}(n, d))= \begin{cases}\sum_{j=1}^{\frac{d}{2}} \frac{n}{n-j}\left(\begin{array}{c}
n-j \\
j
\end{array}\right)\left(\begin{array}{c}
j \\
k-j
\end{array}\right) & \text { if d is even } \\
\sum_{j=1}^{\frac{d+1}{2}} \frac{k+1}{j}\left(\begin{array}{c}
n-j \\
j-1
\end{array}\right)\left(\begin{array}{c}
j \\
k+1-j
\end{array}\right) & \text { if d is odd },\end{cases}
$$

with the usual convention that $\left(\begin{array}{c}n \\ m\end{array}\right)=0$ if $n<m$ or $m<0$. 
Since these formulas are essential ingredient in this paper, we shall include a (shortened) proof for completeness, making use of Shephard's method. In fact, Shephard [7] gave a simple characterization for the faces of $\mathrm{CP}(n, d)$, which generalizes Gale's evenness condition [2] that determines the facets of $\mathrm{CP}(n, d)$. The characterization will be described in the next section (Theorem 2.1). Moreover, Kaibel and Waßmer [4] derived the automorphism group of $\mathrm{CP}(n, d)$.

Theorem 1.2. ([4]) The combinatorial automorphism group of $\mathrm{CP}(n, d)$ is isomorphic to one of the following groups:

\begin{tabular}{l|ccc} 
& $n=d+1$ & $n=d+2$ & $n \geqslant d+3$ \\
\hline$d$ even & $S_{n}$ & $S_{\frac{n}{2}} \mathrm{Wr} \mathbb{Z}_{2}$ & $D_{n}$ \\
$d$ odd & $S_{n}$ & $S_{\left\lceil\frac{n}{2}\right\rceil} \times S_{\left\lfloor\frac{n}{2}\right\rfloor}$ & $\mathbb{Z}_{2} \times \mathbb{Z}_{2}$
\end{tabular}

where $S_{n}$ is the symmetric group of order $n$ and $D_{n}$ is the dihedral group of order $n$.

For the detail of wreath product $S_{\frac{n}{2}}$ wr $\mathbb{Z}_{2}$, we refer the readers to [4]. Consider the cyclic group $C=\mathbb{Z}_{n}$, generated by $c=(1,2 \ldots, n)$, acting on $\operatorname{CP}(n, d)$ by cyclic translation of the vertices, according to the order on the curve $\gamma$. By Theorem 1.2 (or Gale's evenness condition), it turns out that the cyclic group $C$ is an automorphism subgroup of $\mathrm{CP}(n, d)$ if and only if either $n=d+1$ or $d$ is even. One of the main results in this paper is to prove the CSP for faces of $\operatorname{CP}(n, d)$ for even $d$, under $C$-action. Along with a natural $q$-analogue of face number, we are able to state this result. Here we use the notation

$$
\left[\begin{array}{c}
n \\
i
\end{array}\right]_{q}:=\frac{[n] !_{q}}{[i] !_{q}[n-i] !_{q}}
$$

where $[n] !_{q}=[1]_{q}[2]_{q} \cdots[n]_{q}$ and $[i]_{q}=1+q+\cdots+q^{i-1}$. For even $d$ and $1 \leqslant k \leqslant d$, we define

$$
F(n, d, k ; q)=\sum_{j=1}^{\frac{d}{2}} \frac{[n]_{q}}{[n-j]_{q}}\left[\begin{array}{c}
n-j \\
j
\end{array}\right]_{q}\left[\begin{array}{c}
j \\
k-j
\end{array}\right]_{q},
$$

with the usual convention that $\left[\begin{array}{c}n \\ m\end{array}\right]_{q}=0$ if $n<m$ or $m<0$. Clearly, $F(n, d, k ; 1)=$ $f_{k-1}(\mathrm{CP}(n, d))$.

Theorem 1.3. For even $d$ and $1 \leqslant k \leqslant d$, let $X$ be the set of $(k-1)$-faces of $\operatorname{CP}(n, d)$, let $X(q)=F(n, d, k ; q)$ be the polynomial defined in $E q$. (2), and let $C=\mathbb{Z}_{n}$ act on $X$ by cyclic translation of the vertices. Then the triple $(X, X(q), C)$ exhibits the cyclic sieving phenomenon.

For odd $d$, the cyclic group $C$ is not an automorphism subgroup of $\operatorname{CP}(n, d)$ if $n \geqslant$ $d+2$. Inspired by [4], we consider the automorphism subgroup $C^{\prime}$ (resp. $C^{\prime \prime}$ ) of order 2 , generated by $c^{\prime}=(1, n)(2, n-1) \cdots\left(\right.$ resp. $\left.\quad c^{\prime \prime}=(1, n)\right)$, which acts on $\mathrm{CP}(n, d)$ by reversing the order of vertices (resp. by interchanging the first and the last vertices), according to the order on $\gamma$. In an attempt on proving the CSP, we derive the numbers of 
$k$-faces of $\mathrm{CP}(n, d)$ that are invariant under $C^{\prime}$ and $C^{\prime \prime}$, respectively, which are expressible in terms of the formulas in Theorem 1.1. However, so far it lacks a feasible option for the $q$-polynomial $X(q)$. We are interested in a $q$-polynomial that is reasonably neat and serves the purpose of CSP, and we leave it as an open question. For $n=d+2$, from the automorphism group $S_{\left\lceil\frac{n}{2}\right\rceil} \times S_{\left\lfloor\frac{n}{2}\right\rfloor}$ of CP $(n, d)$, we present two instances of CSP, under the group $\mathbb{Z}_{\left\lceil\frac{n}{2}\right\rceil}$ (resp. $\mathbb{Z}_{\left\lfloor\frac{n}{2}\right\rfloor}$ ) that cyclically translates the odd-positioned (resp. evenpositioned) vertices, along with feasible $q$-polynomials.

This paper is organized as follows. We review Shephard's criterion and Gale's evenness condition for cyclic polytopes $\mathrm{CP}(n, d)$ in Section 2. For even $d$, we prove the CSP for faces of $\mathrm{CP}(n, d)$ in Section 3. For odd $d$, we enumerate the faces of $\operatorname{CP}(n, d)$ that are invariant under $C^{\prime}$ and $C^{\prime \prime}$ in Section 4 and Section 5, respectively. The special case $n=d+2$ is discussed in Section 6. A remark regarding the $\operatorname{CSP}$ on $\operatorname{CP}(n, d)$ for odd $d$ is given in Section 7.

\section{Preliminaries}

In this section, we shall review Shephard's characterization for faces of $\mathrm{CP}(n, d)$ and Gale's evenness condition for facets. Based on these results, we include a proof of Theorem 1.1 for completeness.

\subsection{A characterization for faces}

For convenience, let $[n]:=\{1,2 \ldots, n\}$ be the set of vertices of $\operatorname{CP}(n, d)$, numbered according to the order on the curve $\gamma$. For a nonempty subset $U \subseteq[n]$, we associate $U$ with an $(1 \times n)$-array having a star ' $*$ ' at the $i$ th entry if $i \in U$ and a $d o t$ '.' otherwise. In such an array, every maximal segment of consecutive stars is called a block. A block containing the star at entry 1 or $n$ is a border block, and the other ones are inner blocks. For example, the array associated with the face $U=\{1,3,4,7,8,9\}$ of $\mathrm{CP}(9,7)$ is shown in Figure 1, with an inner block $\{3,4\}$ and border blocks $\{1\}$ and $\{7,8,9\}$. A block will be called even or odd according to the parity of its size.

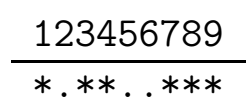

Figure 1: The array associated with the face $U=\{1,3,4,7,8,9\}$ of $\mathrm{CP}(9,7)$.

The following criterion for determining the faces of $\mathrm{CP}(n, d)$ was given by Shephard $[7]$.

Theorem 2.1. For $1 \leqslant k \leqslant d$, a subset $U \subseteq[n]$ is the set of vertices of a $(k-1)$-face of $\mathrm{CP}(n, d)$ if and only if $|U|=k$ and its associated array contains at most $d-k$ odd inner blocks. 
Note that the case $k=d$ in Theorem 2.1 is Gale's evenness condition for determining the facets of $\operatorname{CP}(n, d)$. From this condition, it follows that the cyclic group $C=\mathbb{Z}_{n}$ is an automorphism subgroup of $\mathrm{CP}(n, d)$ only if $n=d+1$ or $d$ is even. Under $C$-action, the face-orbit containing $U$ can be obtained from the associated array simply by shifting the elements cyclically. For example, take $(n, d, k)=(8,4,4)$. As shown in Figure 2, there are twenty facets in $\mathrm{CP}(8,4)$. These facets are partitioned into three orbits, two of which are free orbits and the other one has a stabilizer of order 2. By Theorem 1.3, note that $X(q) \equiv 3+2 q+3 q^{2}+2 q^{3}+3 q^{4}+2 q^{5}+3 q^{6}+2 q^{7}\left(\bmod q^{8}-1\right)$.

\begin{tabular}{|c|c|c|}
\hline 12345678 & 12345678 & 12345678 \\
\hline$* * * *$ & $* * . * * \ldots$ & $* * \ldots * *$ \\
\hline .****. & $. * * . * * \ldots$ &.$* * \ldots * *$ \\
\hline$\ldots * * * *$ & $\ldots * * . * *$ & $\ldots * * \ldots * *$ \\
\hline$\ldots * * * *$ & $\ldots * * . * *$ & $* \ldots * * \ldots *$ \\
\hline$\ldots \ldots * * * *$ & $* \ldots * * . \ldots$ & \\
\hline$* \ldots \ldots * *$ & $* * \ldots * *$. & \\
\hline$* * \ldots * * *$ &.$* * \ldots * *$ & \\
\hline$* * * \ldots \ldots *$ & $* \ldots * \ldots *$ & \\
\hline
\end{tabular}

Figure 2: The orbits for the facets of $\mathrm{CP}(8,4)$ under $\mathbb{Z}_{8}$-action.

\subsection{The enumeration of faces}

Let $A(n, k, s)$ be the set of $(1 \times n)$-arrays with $k$ stars and $s$ odd inner blocks. By Theorem 2.1, we have

$$
f_{k-1}(\mathrm{CP}(n, d))=\sum_{s=0}^{d-k}|A(n, k, s)| .
$$

For enumerative purpose, each array is oriented to form an $n$-cycle, in numerical order clockwise. The $n$-cycles can be viewed as graphs with vertex set $[n]$ colored in black and white such that a vertex is black (resp. white) if the corresponding element is a star (resp. dot). Note that the border blocks of an array become consecutive in the cycle, so by a block of a cycle we mean a maximal sector of black vertices that corresponds to an inner block or the union of the border blocks of the array.

Let $B(n, k, s)$ be the set of such $n$-cycles with $k$ black vertices and $s$ odd blocks, where $s$ and $k$ have the same parity necessarily. Note that each cycle $\beta \in B(n, k, s)$ associates with a unique array $\alpha$ by cutting the edge between vertices 1 and $n$. It follows from $s \equiv k$ $(\bmod 2)$ that

$$
|B(n, k, s)|=|A(n, k, s)|+|A(n, k, s-1)| .
$$

Note that if $\alpha \in A(n, k, s-1)$ then the union of the border blocks of $\alpha$ is of odd size. In this case, $\beta$ has one more odd block than $\alpha$. 
Proposition 2.2. For $1 \leqslant k<n$ and $0 \leqslant s \leqslant k$, we have

(i) $|B(n, 2 i, 0)|=\frac{n}{n-i}\left(\begin{array}{c}n-i \\ i\end{array}\right), \quad$ for $1 \leqslant i<\frac{n}{2}$.

(ii) $|B(n, k, s)|=\frac{n}{n-j}\left(\begin{array}{c}n-j \\ j\end{array}\right)\left(\begin{array}{l}j \\ s\end{array}\right)$, where $j=\frac{k+s}{2}$.

Proof. (i) For each $\beta \in B(n, 2 i, 0)$, we partition the $2 i$ black vertices of $\beta$ into $i$ adjacent pairs. Each of these pairs is connected by a blue edge and the other $n-i$ edges of $\beta$ are colored red. We count the number of ordered pairs $(\beta, e)$ such that $e$ is an edge of $\beta$ and $\beta-e$ is a path of length $n-1$ with no odd blocks, where $\beta-e$ is obtained from $\beta$ by cutting $e$.

For each $\beta \in B(n, 2 i, 0)$, the edge $e$ can be any one of the $n-i$ red edges. On the other hand, given a path $\pi$ of length $n-1$ with $i$ adjacent pairs $p_{1}, \ldots, p_{i}$ of black vertices, let $y_{j}$ be the number of white vertices between $p_{j-1}$ and $p_{j}$, for $2 \leqslant j \leqslant i$, and let $y_{1}$ (resp. $y_{i+1}$ ) be the number of white vertices before $p_{1}$ (resp. after $p_{i}$ ). Then the possibilities of $\pi$ is the number of nonnegative solutions of the equation $y_{1}+\cdots+y_{i+1}=n-2 i$, which is given by $\left(\begin{array}{c}n-i \\ i\end{array}\right)$. Moreover, there are $n$ ways to label the vertices of $\pi$ cyclically by $[n]$. After adding an edge $e$ that connects both ends, we turn $\pi$ into an $n$-cycle $\pi+e \in B(n, 2 i, 0)$. Hence

$$
|B(n, 2 i, 0)| \cdot(n-i)=n \cdot\left(\begin{array}{c}
n-i \\
i
\end{array}\right) .
$$

The assertion (i) follows.

Given a $\beta \in B(n, k, s)$, each block of $\beta$ is followed by a unique immediate white vertex, called successor, in numerical order. We enumerate the ordered pairs $(\beta, S)$ such that the set $S$ consists of the successors of the $s$ odd blocks of $\beta$. Coloring in black the vertices in $S$ leads to a cycle in $B(n, k+s, 0)$. On the other hand, for any $\beta^{\prime} \in B(n, k+s, 0)$, there are $\frac{k+s}{2}$ pairs of adjacent black vertices. Let $S$ be the set consisting of the second vertex in any $s$ of these pairs. Coloring in white the vertices in $S$ recovers a cycle in $B(n, k, s)$. Hence we have

$$
|B(n, k, s)|=\left(\begin{array}{c}
\frac{k+s}{2} \\
s
\end{array}\right)|B(n, k+s, 0)|
$$

The assertion (ii) follows from (i).

Now, we are able to prove Theorem 1.1.

Proof of Theorem 1.1. For even $d$ and $1 \leqslant k \leqslant d$, it follows from Eq. (3), (4) and Proposition 2.2(ii) that the number of $(k-1)$-faces is

$$
\sum_{s=0}^{d-k}|A(n, k, s)|=\sum_{\substack{s=0 \\
s \equiv k(\bmod 2)}}^{d-k}|B(n, k, s)|=\sum_{j=1}^{\frac{d}{2}} \frac{n}{n-j}\left(\begin{array}{c}
n-j \\
j
\end{array}\right)\left(\begin{array}{c}
j \\
k-j
\end{array}\right)
$$

as required. (Note that the terms corresponding to $1 \leqslant j<\left\lceil\frac{k}{2}\right\rceil$ in the summation are zero.) 
For odd $d$ and $1 \leqslant k \leqslant d$, each array $\alpha$ that corresponds to a face in $\operatorname{CP}(n, d)$ is oriented to form an $(n+1)$-cycle $\beta$ by adding a black vertex, labeled by $n+1$, between vertices 1 and $n$. We observe that $\beta \in B(n+1, k+1, s)$ if and only if $\alpha \in A(n, k, s-1) \cup A(n, k, s)$, where $s \equiv k+1(\bmod 2)$. We count the number of ordered pairs $(\beta, e)$, where $\beta \in B(n+1, k+1, s)$ and $e$ is an edge of $\beta$ such that $\beta-e$ is a path of length $n$ with a black vertex at the end.

Given a $\beta \in B(n+1, k+1, s)$, the edge $e$ can be any one of the $k+1$ edges in $\beta$ the second vertex of which is black. On the other hand, for any $\pi \in A(n, k, s-1) \cup A(n, k, s)$, we add a black vertex at the end of $\pi$ and label these vertices cyclically by $[n+1]$. After adding an edge that connects both ends, we turn the new path into an $(n+1)$-cycle in $B(n+1, k+1, s)$. Hence

$$
|B(n+1, k+1, s)| \cdot(k+1)=(|A(n, k, s-1)|+|A(n, k, s)|) \cdot(n+1) .
$$

By Proposition 2.2(ii), the number of $(k-1)$-faces is

$$
\sum_{s=0}^{d-k}|A(n, k, s)|=\sum_{\substack{s=0 \\
s \equiv k+1(\bmod 2)}}^{d-k} \frac{k+1}{n+1} \cdot|B(n+1, k+1, s)|=\sum_{j=1}^{\frac{d+1}{2}} \frac{k+1}{j}\left(\begin{array}{c}
n-j \\
j-1
\end{array}\right)\left(\begin{array}{c}
j \\
k+1-j
\end{array}\right) .
$$

This completes the proof of Theorem 1.1.

\section{The CSP for faces of $\mathrm{CP}(n, d)$ for even $d$}

In this section, we shall prove Theorem 1.3 by verifying the condition (1) mentioned in the introduction. The following $q$-Lucas theorem is helpful in evaluating $X(q)$ at primitive roots of unity (see [6, Theorem 2.2]).

Lemma 3.1. (q-Lucas Theorem) Let $\omega$ be a primitive rth root of unity. If $n=$ ar $+b$ and $k=c r+d$, where $0 \leqslant b, d \leqslant r-1$, then

$$
\left[\begin{array}{l}
n \\
k
\end{array}\right]_{q=\omega}=\left(\begin{array}{l}
a \\
c
\end{array}\right)\left[\begin{array}{l}
b \\
d
\end{array}\right]_{q=\omega}
$$

Proof of Theorem 1.3. For $r \geqslant 2$ a divisor of $n$, let $\omega$ be a primitive $r$ th root of unity and let $C_{r}$ be the subgroup of order $r$ of $C$. Let $d=2 t$. First, we claim that

$$
[F(n, 2 t, k ; q)]_{q=\omega}= \begin{cases}\sum_{i=1}^{\left\lfloor\frac{t}{r}\right\rfloor} \frac{n}{n-i r}\left(\begin{array}{c}
\frac{n}{r}-i \\
i
\end{array}\right)\left(\begin{array}{c}
i \\
\frac{k}{r}-i
\end{array}\right) & \text { if } r \mid k \\
\text { otherwise. }\end{cases}
$$

Since $r \mid n$, it is straightforward to prove that

$$
\lim _{q \rightarrow \omega} \frac{[n]_{q}}{[n-j]_{q}}= \begin{cases}\frac{n}{n-j} & \text { if } r \mid j \\ 0 & \text { otherwise. }\end{cases}
$$


By $q$-Lucas Theorem, for $r \mid n$ and $r \mid j$, we have

$$
\left[\begin{array}{c}
n-j \\
j
\end{array}\right]_{q=\omega}=\left(\begin{array}{c}
\frac{n-j}{r} \\
\frac{j}{r}
\end{array}\right) \text { and }\left[\begin{array}{c}
j \\
k-j
\end{array}\right]_{q=\omega}= \begin{cases}\left(\begin{array}{c}
\frac{j}{r} \\
\frac{k-j}{r}
\end{array}\right) & \text { if } r \mid k \\
0 & \text { otherwise. }\end{cases}
$$

Then evaluate Eq. (2) at $q=\omega$ and take Eq. (6), (7) into account. This proves Eq. (5).

Next, we enumerate the $(k-1)$-faces of $\mathrm{CP}(n, 2 t)$ that are invariant under $C_{r}$. Let $V(n, k, s, r) \subseteq A(n, k, s)$ (resp. $W(n, k, s, r) \subseteq B(n, k, s)$ ) be the subset of arrays (resp. cycles) that are $C_{r}$-invariant. It is clear that $r \mid k$ and $r \mid s$ if $W(n, k, s, r)$ is nonempty. Moreover, it follows from a set version of Eq. (4) that

$$
|W(n, k, s, r)|=|V(n, k, s, r)|+|V(n, k, s-1, r)| .
$$

Given a $\beta \in W(n, k, s, r)$, we partition $\beta$ into $r$ identical sectors $\mu_{1}, \ldots, \mu_{r}$, where $\mu_{i}$ consists of the vertices $\left\{\frac{n(i-1)}{r}+1, \ldots, \frac{n i}{r}\right\}$. Let $\widehat{\mu}_{1}$ be the cycle obtained from $\mu_{1}$ by adding an edge that connects vertices 1 and $\frac{n}{r}$. We observe that $\widehat{\mu}_{1} \in B\left(\frac{n}{r}, \frac{k}{r}, \frac{s}{r}\right)$. On the other hand, given an $\widehat{\mu}^{\prime} \in B\left(\frac{n}{r}, \frac{k}{r}, \frac{s}{r}\right)$, let $\mu^{\prime}$ be the path obtained from $\widehat{\mu}^{\prime}$ by cutting the edge between vertices 1 and $\frac{n}{r}$. One can recover an $n$-cycle $\beta^{\prime} \in W(n, k, s, r)$ from the path $\mu^{\prime} \cdots \mu^{\prime}$ formed by a concatenation of $r$ copies of $\mu^{\prime}$. This establishes a bijection between $W(n, k, s, r)$ and $B\left(\frac{n}{r}, \frac{k}{r}, \frac{s}{r}\right)$. Hence the number of $(k-1)$-faces of $\operatorname{CP}(n, d)$ that are $C_{r}$-invariant is given by

$$
\begin{aligned}
\sum_{s=0}^{2 t-k}|V(n, k, s, r)| & =\sum_{\substack{s=0 \\
s \equiv k(\bmod 2)}}^{2 t-k}|W(n, k, s, r)| \\
& =\sum_{\substack{s=0 \\
s \equiv k \bmod 2) \\
r|k, r| s}}^{2 t-k}\left|B\left(\frac{n}{r}, \frac{k}{r}, \frac{s}{r}\right)\right| \\
& =\sum_{i=1}^{\left\lfloor\frac{t}{r}\right\rfloor} \frac{n}{n-i r}\left(\begin{array}{c}
\frac{n}{r}-i \\
i
\end{array}\right)\left(\begin{array}{c}
i \\
\frac{k}{r}-i
\end{array}\right)
\end{aligned}
$$

if $r \mid k$ and 0 otherwise, which agrees with Eq. (5). This completes the proof of Theorem 1.3 .

\section{The $C^{\prime}$-invariant faces of $\mathrm{CP}(n, d)$}

In this section, we consider the cyclic group $C^{\prime}$ of order 2 , generated by $c^{\prime}=(1, n)(2, n-$ $1) \cdots$, acting on $\mathrm{CP}(n, d)$ by carrying vertex $i$ to vertex $n+1-i(1 \leqslant i \leqslant n)$. Under $C^{\prime}$-action, each array that corresponds to a face is carried to another array by flipping about the central line of the array. We shall enumerate the faces of $\operatorname{CP}(n, d)$ that are invariant under $C^{\prime}$-action. We treat the cases of odd $d$ and even $d$ separately. 
The counting formulas in Theorem 1.1 for the face number of $\operatorname{CP}(n, d)$ are helpful in enumerating the set $A(n, k, s)$ of $(1 \times n)$-arrays with $k$ stars and $s$ odd inner blocks. For $1 \leqslant k \leqslant d$, we define

$$
\begin{aligned}
& f(n, d, k)=\sum_{j=1}^{\frac{d}{2}} \frac{n}{n-j}\left(\begin{array}{c}
n-j \\
j
\end{array}\right)\left(\begin{array}{c}
j \\
k-j
\end{array}\right) \quad \text { for even } d \\
& g(n, d, k)=\sum_{j=1}^{\frac{d+1}{2}} \frac{k+1}{j}\left(\begin{array}{c}
n-j \\
j-1
\end{array}\right)\left(\begin{array}{c}
j \\
k+1-j
\end{array}\right) \text { for odd } d .
\end{aligned}
$$

Proposition 4.1. For $m \geqslant 0$, the following equations hold.

(i) We have

$$
\sum_{s=0}^{m}|A(n, k, s)|= \begin{cases}f(n, k+m, k) & \text { if } k+m \text { is even } \\ g(n, k+m, k) & \text { if } k+m \text { is odd. }\end{cases}
$$

(ii) We have

$$
|A(n, k, m)|= \begin{cases}f(n, k+m, k)-g(n, k+m-1, k) & \text { if } k+m \text { is even } \\ g(n, k+m, k)-f(n, k+m-1, k) & \text { if } k+m \text { is odd }\end{cases}
$$

with the assumption that $f(n, i, j)=g(n, i, j)=0$ for $i<j$.

Proof. The assertion (i) follows immediately from Theorem 1.1 and Eq. (3). The assertion (ii) is obtained from (i) by computing $\sum_{s=0}^{m}|A(n, k, s)|-\sum_{s=0}^{m-1}|A(n, k, s)|$.

For the faces of $\operatorname{CP}(n, d)$ under $C^{\prime}$-action, we have the following enumerative results.

Theorem 4.2. For odd $d$ and $1 \leqslant k \leqslant d$, the number $h_{(n, d, k-1)}$ of $(k-1)$-faces of $\operatorname{CP}(n, d)$ that are $C^{\prime}$-invariant is given as follows.

(i) If $n$ is even, then

$$
h_{(n, d, k-1)}= \begin{cases}0 & \text { if } k \text { is odd } \\ f\left(\frac{n}{2}, \frac{d-1}{2}, \frac{k}{2}\right) & \text { if } k \text { is even, } d \equiv 1(\bmod 4) \\ g\left(\frac{n}{2}, \frac{d-1}{2}, \frac{k}{2}\right) & \text { if } k \text { is even, } d \equiv 3(\bmod 4) .\end{cases}
$$

(ii) If $n$ is odd, then

$$
h_{(n, d, k-1)}= \begin{cases}g\left(\frac{n-1}{2}, \frac{d-3}{2}, \frac{k-1}{2}\right) & \text { if } k \text { is odd, } d \equiv 1(\bmod 4) \\ f\left(\frac{n-1}{2}, \frac{d-3}{2}, \frac{k-1}{2}\right) & \text { if } k \text { is odd, } d \equiv 3(\bmod 4) \\ f\left(\frac{n+1}{2}, \frac{d-1}{2}, \frac{k}{2}\right)-g\left(\frac{n-1}{2}, \frac{d-3}{2}, \frac{k}{2}-1\right) & \text { if } k \text { is even, } d \equiv 1(\bmod 4) \\ g\left(\frac{n+1}{2}, \frac{d-1}{2}, \frac{k}{2}\right)-f\left(\frac{n-1}{2}, \frac{d-3}{2}, \frac{k}{2}-1\right) & \text { if } k \text { is even, } d \equiv 3(\bmod 4),\end{cases}
$$

with the assumption that $f(n, i, j)=g(n, i, j)=0$ for $i<j$ and $f(n, m, 0)=$ $g(n, m, 0)=1$ for all $m \geqslant 0$. 
Proof. Let $U(n, k, s) \subseteq A(n, k, s)$ be the set of arrays that are invariant under $C^{\prime}$-action.

(i) For even $n$, given an $\alpha \in A(n, k, s)$, the central line $L$ of $\alpha$ lies between vertices $\frac{n}{2}$ and $\frac{n}{2}+1$. Let $\alpha=\left(\alpha_{1}, \alpha_{2}\right)$ be cut in half, where $\alpha_{1}$ is on the set $\left\{1, \ldots, \frac{n}{2}\right\}$ and $\alpha_{2}$ is on the set $\left\{\frac{n}{2}+1, \ldots, n\right\}$. Note that $\alpha \in U(n, k, s)$ (i.e., $C^{\prime}$-invariant) if and only if $\alpha$ is symmetric with respect to $L$, in which case $\alpha_{1}, \alpha_{2} \in A\left(\frac{n}{2}, \frac{k}{2}, \frac{s}{2}\right)$, where $s \equiv k \equiv 0(\bmod 2)$ necessarily. Hence by Proposition 4.1, the number of $(k-1)$-faces that are $C^{\prime}$-invariant is

$$
\begin{aligned}
\sum_{\substack{s=0 \\
s \equiv k \equiv 0(\bmod 2)}}^{d-1-k}|U(n, k, s)| & =\sum_{\substack{s=0 \\
s \equiv k \equiv 0(\bmod 2)}}^{\frac{d-1-k}{2}}\left|A\left(\frac{n}{2}, \frac{k}{2}, \frac{s}{2}\right)\right| \\
& =\sum_{s^{\prime}=0}^{d-1-k}\left|A\left(\frac{n}{2}, \frac{k}{2}, s^{\prime}\right)\right| \\
& = \begin{cases}f\left(\frac{n}{2}, \frac{d-1}{2}, \frac{k}{2}\right) & \text { if } d \equiv 1(\bmod 4) \\
g\left(\frac{n}{2}, \frac{d-1}{2}, \frac{k}{2}\right) & \text { if } d \equiv 3(\bmod 4) .\end{cases}
\end{aligned}
$$

(ii) For odd $n$, given an $\alpha \in A(n, k, s)$, the central line $L$ passes through vertex $\frac{n+1}{2}$. Let $\alpha=\left(\alpha_{1}, \frac{n+1}{2}, \alpha_{2}\right)$, where $\alpha_{1}$ is on the set $\left\{1, \ldots, \frac{n-1}{2}\right\}$ and $\alpha_{2}$ is on the set $\left\{\frac{n+3}{2}, \ldots, n\right\}$. There are two cases.

Case I. $k$ is odd. Then $\alpha \in U(n, k, s)$ if and only if there is a star at the middle entry $\frac{n+1}{2}$ and $\alpha_{1} \cup \alpha_{2}$ is symmetric with respect to $L$, in which case $\alpha_{1}, \alpha_{2} \in A\left(\frac{n-1}{2}, \frac{k-1}{2}, \frac{s-1}{2}\right)$, where $k \equiv s \equiv 1(\bmod 2)$ necessarily. Hence the number of $(k-1)$-faces that are $C^{\prime}$ invariant is

$$
\begin{aligned}
\sum_{\substack{s=1 \\
s \equiv k \equiv 1(\bmod 2)}}^{d-1-k}|U(n, k, s)| & =\sum_{\substack{s=1 \\
s \equiv k \equiv 1(\bmod 2)}}^{\frac{d-1-k}{d-k}} \sum_{s^{\prime}=0}\left|A\left(\frac{n-1}{2}, \frac{k-1}{2}, \frac{s-1}{2}\right)\right| \\
& = \begin{cases}g\left(\frac{n-1}{2}, \frac{d-3}{2}, \frac{k-1}{2}\right) & \text { if } d \equiv 1(\bmod 4) \\
f\left(\frac{n-1}{2}, \frac{d-3}{2}, \frac{k-1}{2}\right) & \text { if } d \equiv 3(\bmod 4) .\end{cases}
\end{aligned}
$$

Case II. $k$ is even. Then $\alpha \in U(n, k, s)$ if and only if there is a dot at the middle entry $\frac{n+1}{2}$ and $\alpha_{1} \cup \alpha_{2}$ is symmetric with respect to $L$. To compute $|U(n, k, s)|$, let $\alpha_{1}^{\prime}=\alpha_{1} \cup\{$.$\} be the array on the set \left\{1, \ldots, \frac{n+1}{2}\right\}$ obtained from $\alpha$ by adding a dot at $\frac{n+1}{2}$. Then $\alpha_{1}^{\prime}$ is a member of $A\left(\frac{n+1}{2}, \frac{k}{2}, \frac{s}{2}\right)$ such that there is a dot at the end. Note that there are $\left|A\left(\frac{n-1}{2}, \frac{k}{2}-1, \frac{s}{2}\right)\right|$ members in $A\left(\frac{n+1}{2}, \frac{k}{2}, \frac{s}{2}\right)$ with a star at the end since $\pi \in A\left(\frac{n-1}{2}, \frac{k}{2}-1, \frac{s}{2}\right)$ if and only if $\pi \cup\{*\}$ is a member of $A\left(\frac{n+1}{2}, \frac{k}{2}, \frac{s}{2}\right)$ such that there is 
a star at the end. Hence the number of $(k-1)$-faces that are $C^{\prime}$-invariant is

$$
\begin{aligned}
\sum_{\substack{s=0 \\
s \equiv k \equiv 0(\bmod 2)}}^{d-1-k}|U(n, k, s)| & =\sum_{\substack{s=0 \\
s \equiv k \equiv(\bmod 2)}}^{\frac{d-1-k}{2}}\left|A\left(\frac{n+1}{2}, \frac{k}{2}, \frac{s}{2}\right)\right|-\left|A\left(\frac{n-1}{2}, \frac{k}{2}-1, \frac{s}{2}\right)\right| \\
& =\sum_{s^{\prime}=0}^{d-1}\left|A\left(\frac{n+1}{2}, \frac{k}{2}, s^{\prime}\right)\right|-\left|A\left(\frac{n+1}{2}, \frac{k}{2}-1, s^{\prime}\right)\right| \\
& = \begin{cases}f\left(\frac{n+1}{2}, \frac{d-1}{2}, \frac{k}{2}\right)-g\left(\frac{n-1}{2}, \frac{d-3}{2}, \frac{k}{2}-1\right) & \text { if } d \equiv 1(\bmod 4) \\
g\left(\frac{n+1}{2}, \frac{d-1}{2}, \frac{k}{2}\right)-f\left(\frac{n-1}{2}, \frac{d-3}{2}, \frac{k}{2}-1\right) & \text { if } d \equiv 3(\bmod 4) .\end{cases}
\end{aligned}
$$

The proof is completed.

For example, take $(n, d, k)=(9,5,4)$. There are $g(9,5,4)=75$ faces of dimension 3 in $\mathrm{CP}(9,5)$, whose arrays contain 4 stars and at most 1 odd inner block. By Theorem 4.2(ii), the number of 3 -faces that are $C^{\prime}$-invariant is $f(5,2,2)-g(4,1,1)=3$, which are shown in Figure 3(a). Moreover, take $(n, d, k)=(9,7,4)$. There are $g(9,7,4)=125$ faces of dimension 3 in $\mathrm{CP}(9,7)$, whose arrays contain 4 stars and at most 3 odd inner blocks. By Theorem 4.2(ii), the number of 3 -faces that are $C^{\prime}$-invariant is $g(5,3,2)-f(4,2,1)=5$, which are shown in Figure 3(b).

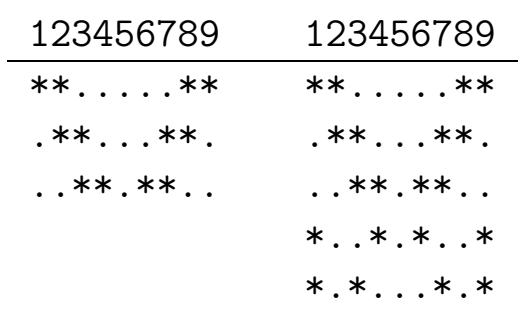

(a)

(b)

Figure 3: The $C^{\prime}$-invariant 3-faces of $\mathrm{CP}(9,5)$ and $\mathrm{CP}(9,7)$, respectively.

By the same argument as in the proof of Theorem 4.2, we obtain the following enumerative results for even $d$.

Theorem 4.3. For even $d$ and $1 \leqslant k \leqslant d$, the number $h_{(n, d, k-1)}$ of $(k-1)$-faces of $\mathrm{CP}(n, d)$ that are $C^{\prime}$-invariant is given as follows.

(i) If $n$ is even, then

$$
h_{(n, d, k-1)}= \begin{cases}0 & \text { if } k \text { is odd } \\ f\left(\frac{n}{2}, \frac{d}{2}, \frac{k}{2}\right) & \text { if } k \text { is even, } d \equiv 0(\bmod 4) \\ g\left(\frac{n}{2}, \frac{d}{2}, \frac{k}{2}\right) & \text { if } k \text { is even, } d \equiv 2(\bmod 4) .\end{cases}
$$


(ii) If $n$ is odd, then

$$
h_{(n, d, k-1)}= \begin{cases}g\left(\frac{n-1}{2}, \frac{d-2}{2}, \frac{k-1}{2}\right) & \text { if } k \text { is odd, } d \equiv 0(\bmod 4) \\ f\left(\frac{n-1}{2}, \frac{d-2}{2}, \frac{k-1}{2}\right) & \text { if } k \text { is odd, } d \equiv 2(\bmod 4) \\ f\left(\frac{n+1}{2}, \frac{d}{2}, \frac{k}{2}\right)-g\left(\frac{n-1}{2}, \frac{d-2}{2}, \frac{k}{2}-1\right) & \text { if } k \text { is even, } d \equiv 0(\bmod 4) \\ g\left(\frac{n+1}{2}, \frac{d}{2}, \frac{k}{2}\right)-f\left(\frac{n-1}{2}, \frac{d-2}{2}, \frac{k}{2}-1\right) & \text { if } k \text { is even, } d \equiv 2(\bmod 4),\end{cases}
$$

with the assumption that $f(n, i, j)=g(n, i, j)=0$ for $i<j$ and $f(n, m, 0)=$ $g(n, m, 0)=1$ for all $m \geqslant 0$.

\section{The $C^{\prime \prime}$-invariant faces of $\mathrm{CP}(n, d)$}

In this section, we enumerate the faces of $\mathrm{CP}(n, d)$ that are invariant under the automorphism $c^{\prime \prime}=(1, n)$ that interchanges vertices 1 and $n$.

Theorem 5.1. For odd $d$ and $1 \leqslant k \leqslant d$, the number $z_{(n, d, k-1)}$ of $(k-1)$-faces of $\mathrm{CP}(n, d)$ that are $C^{\prime \prime}$-invariant is given by

$$
z_{(n, d, k-1)}=g(n, d, k)-2 f(n-1, d-1, k-1)+2 g(n-2, d-2, k-2),
$$

with the assumption that $f(n, m, 0)=g(n, m, 0)=1$ for all $m \geqslant 0$, and $f(n, m,-1)=$ $g(n, m,-1)=0$ for all $m \geqslant-1$.

Proof. Given an $\alpha \in A(n, k, s)$, let $\alpha=v_{1} \cdots v_{n}$, where $v_{i}$ is the element at the $i$ th entry of $\alpha$. Note that $\alpha$ is $C^{\prime \prime}$-invariant if and only if $v_{1}$ and $v_{n}$ are identical (i.e., both are either stars or dots). Let $Y(n, k, s)$ and $Z(n, k, s)$ be subsets of $A(n, k, s)$ such that $\alpha \in Y(n, k, s)$ if $\left(v_{1}, v_{n}\right)=\left(\right.$ star, dot), and $\alpha \in Z(n, k, s)$ if $\left(v_{1}, v_{n}\right)=$ (dot, star). Clearly, $|Y(n, k, s)|=|Z(n, k, s)|$ (by a reverse vertex-order). To compute $|Y(n, k, s)|$, we observe that $\alpha \in Y(n, k, s)$ if and only if the segment $v_{2} \cdots v_{n}$ is a member of $A(n-1, k-1, s)$ such that there is a dot at the end. Moreover, there are $|A(n-2, k-2, s)|$ members in $A(n-1, k-1, s)$ with a star at the end since the segment $\pi=v_{2} \cdots v_{n-1} \in A(n-2, k-2, s)$ if and only if $\pi \cup\{*\}$ is a member of $A(n-1, k-1, s)$ such that there is a star at the end. Then we have

$$
|Y(n, k, s)|=|A(n-1, k-1, s)|-|A(n-2, k-2, s)| .
$$

Hence the number of $(k-1)$-faces that are $C^{\prime \prime}$-invariant is

$$
\begin{aligned}
z_{(n, d, k-1)} & =\sum_{s=0}^{d-k}|A(n, k, s)-Y(n, k, s)-Z(n, k, s)| \\
& =\sum_{s=0}^{d-k}|A(n, k, s)|-2|A(n-1, k-1, s)|+2|A(n-2, k-2, s)| .
\end{aligned}
$$

By Proposition 4.1(i), the assertion follows. 
We remark that the cyclic group $C^{\prime \prime}$ is not an automorphism subgroup of $\mathrm{CP}(n, d)$ if $d$ is even. To see this, for example, a facet $U=\{1,2,3,4\}$ of $\mathrm{CP}(7,4)$ is carried to the vertex set $\{2,3,4,7\}$ by $c^{\prime \prime}$, which is not a face of $\mathrm{CP}(7,4)$.

\section{The cyclic polytopes $\mathrm{CP}(n, d)$ for $n=d+1$ and $n=$ $d+2$}

In this section, we show instances of CSP on $\mathrm{CP}(n, d)$ for the cases $n=d+1$ and $n=d+2$.

\subsection{The case $n=d+1$.}

For $n=d+1$, the cyclic polytope $\mathrm{CP}(d+1, d)$ is combinatorially equivalent to a regular simplex, whose $(k-1)$-face number is given by

$$
f_{k-1}(\mathrm{CP}(d+1, d))=\left(\begin{array}{c}
d+1 \\
k
\end{array}\right), \quad \text { for } 1 \leqslant k \leqslant d .
$$

For even $d$, Theorem 1.3 gives the CSP for faces of $\operatorname{CP}(d+1, d)$, under the cyclic group $C=\mathbb{Z}_{d+1}$ action. In fact, it is straightforward to prove that this also holds for odd $d$.

Theorem 6.1. For $1 \leqslant k \leqslant d$, let $X$ be the set of $(k-1)$-faces of $\mathrm{CP}(d+1, d)$, let $X(q)=\left[\begin{array}{c}d+1 \\ k\end{array}\right]_{q}$, and let $C=\mathbb{Z}_{d+1}$ act on $X$ by cyclic translation of the vertices. Then the triple $(X, X(q), C)$ exhibits the cyclic sieving phenomenon.

\subsection{The case $n=d+2$.}

For $n=d+2$, the arrays associated with the facets of $\mathrm{CP}(d+2, d)$ contain $d$ stars and 2 dots. By Gale's evenness condition, these two dots, say at entries $i, j$, define an even inner block in the array, which implies that $i$ and $j$ have different parity. Let $[n]_{\text {odd }}:=[n] \cap(2 \mathbb{Z}+1)$ and $[n]_{\text {even }}:=[n] \cap(2 \mathbb{Z})$. According to [4, Proposition 8.5], the cyclic polytope $\mathrm{CP}(d+2, d)$ can be realized as the free sum of two simplices $P$ and $Q$, where $P($ resp. $Q)$ is on the vertex set $[d+2]_{\text {odd }}\left(\right.$ resp. $\left.[d+2]_{\text {even }}\right)$. By Shephard's characterization for faces, we have the following observation.

Proposition 6.2. For $1 \leqslant k \leqslant d$, a subset $U \subseteq[d+2]$ is the set of vertices of a $(k-1)$-face of $\mathrm{CP}(d+2, d)$ if and only if $|U|=k$ and $k-\left\lfloor\frac{d}{2}\right\rfloor \leqslant\left|U \cap[d+2]_{\text {odd }}\right| \leqslant\left\lceil\frac{d}{2}\right\rceil$.

Proof. By Theorem 2.1, $U$ is the vertex set of a $(k-1)$-face if and only if $|U|=k$, and the array associated with $U$ contains at most $d-k$ odd inner blocks. It follows that such an array contains $d+2-k$ dots, at most $d+1-k$ of which are at odd (resp. even) entries, and hence at least one of which is at even (resp. odd) entry. If $j=\left|U \cap[d+2]_{\text {odd }}\right|$, then counting the number of dots in $[d+2]_{\text {odd }}$ leads to $1 \leqslant\left\lceil\frac{d+2}{2}\right\rceil-j \leqslant d+1-k$. Hence $k-\left\lfloor\frac{d}{2}\right\rfloor \leqslant j \leqslant\left\lceil\frac{d}{2}\right\rceil$, as required. 
By Proposition 6.2, we have a simplified expression for the face number of $\mathrm{CP}(d+2, d)$.

Corollary 6.3. For odd $d$ and $1 \leqslant k \leqslant d$, the number $f_{k-1}(\mathrm{CP}(d+2, d))$ of $(k-1)$-faces of $\mathrm{CP}(d+2, d)$ is given by

$$
f_{k-1}(\mathrm{CP}(d+2, d))=\sum_{j=1}^{d+1-k}\left(\begin{array}{c}
\frac{d+3}{2} \\
j
\end{array}\right)\left(\begin{array}{c}
\frac{d+1}{2} \\
d+2-k-j
\end{array}\right),
$$

with the usual convention that $\left(\begin{array}{c}n \\ m\end{array}\right)=0$ if $n<m$ or $m<0$.

Proof. For odd $d,\left|[d+2]_{\text {odd }}\right|=\frac{d+3}{2}$ and $\left|[d+2]_{\text {even }}\right|=\frac{d+1}{2}$. Note that the arrays associated with $(k-1)$-face contain $d+2-k$ dots. We enumerate the $(k-1)$-faces with respect to the number $j$ of dots contained in $[d+2]_{\text {odd }}$, where $1 \leqslant j \leqslant d+1-k$ (by Proposition 6.2 ), and the assertion follows.

According to the automorphism group of $\mathrm{CP}(d+2, d)$, shown in Theorem 1.2, we present instances of CSP for faces of $\mathrm{CP}(d+2, d)$ for odd $d$, with 'artificial' $q$-analogues of face number defined by

$$
\begin{aligned}
P(d, k ; q) & =\sum_{j=1}^{d+1-k}\left[\begin{array}{c}
\frac{d+3}{2} \\
j
\end{array}\right]_{q}\left(\begin{array}{c}
\frac{d+1}{2} \\
d+2-k-j
\end{array}\right) \\
Q(d, k ; q) & =\sum_{j=1}^{d+1-k}\left(\begin{array}{c}
\frac{d+3}{2} \\
d+2-k-j
\end{array}\right)\left[\begin{array}{l}
\frac{d+1}{2} \\
j
\end{array}\right]_{q}
\end{aligned}
$$

Theorem 6.4. For odd $d$ and $1 \leqslant k \leqslant d$, let $X$ be the set of $(k-1)$-faces of $\mathrm{CP}(d+2, d)$. Then the following results hold.

(i) Let $X_{1}(q)=P(d, k ; q)$ and let $C_{1}=\mathbb{Z}_{\frac{d+3}{2}}$ act on $X$ by cyclic translation of the vertex subset $[d+2]_{\text {odd }}$ of $\mathrm{CP}(d+2, d)$. Then the triple $\left(X, X_{1}(q), C_{1}\right)$ exhibits the cyclic sieving phenomenon.

(ii) Let $X_{2}(q)=Q(d, k ; q)$ and let $C_{2}=\mathbb{Z}_{\frac{d+1}{2}}$ act on $X$ by cyclic translation of the vertex subset $[d+2]_{\text {even }}$ of $\mathrm{CP}(d+2, d)$. Then the triple $\left(X, X_{2}(q), C_{2}\right)$ exhibits the cyclic sieving phenomenon.

Proof. For $r \geqslant 2$ a divisor of $\frac{d+3}{2}$, let $\omega$ be a primitive $r$ th root of unity,. By Lemma 3.1, we have

$$
\lim _{q \rightarrow \omega}\left[\begin{array}{c}
\frac{d+3}{2} \\
j
\end{array}\right]_{q}= \begin{cases}\left(\begin{array}{c}
\frac{d+3}{2 r} \\
\frac{j}{r}
\end{array}\right) & \text { if } r \mid j \\
0 & \text { otherwise. }\end{cases}
$$

Hence

$$
\left[X_{1}(q)\right]_{q=\omega}=\sum_{i=1}^{\left\lfloor\frac{d+1-k}{r}\right\rfloor}\left(\begin{array}{c}
\frac{d+3}{2 r} \\
i
\end{array}\right)\left(\begin{array}{c}
\frac{d+1}{2} \\
d+2-k-i r
\end{array}\right) .
$$


Given a $\pi \in X$ with $j$ dots at odd entries. We observe that $\pi$ is invariant under the subgroup $C_{(1, r)}$ of order $r$ of $C_{1}$ if and only if the subarray induced on $[d+2]_{\text {odd }}$ can be partitioned into $r$ identical segments of size $\frac{d+3}{2 r}$, in which case $r$ divides $j$ since each segment contains exactly $\frac{j}{r}$ dots. Hence the number of $(k-1)$-faces that are $C_{(1, r)}$-invariant is given by

$$
\sum_{i=1}^{\left\lfloor\frac{d+1-k}{r}\right\rfloor}\left(\begin{array}{c}
\frac{d+3}{2 r} \\
i
\end{array}\right)\left(\begin{array}{c}
\frac{d+1}{2} \\
d+2-k-i r
\end{array}\right),
$$

which agrees with Eq. (10). The proves the assertion (i).

The assertion (ii) can be proved by a similar argument.

For example, take $(d, k)=(5,3)$ and let $\mathbb{Z}_{4}$ act on $\mathrm{CP}(7,5)$ by cyclic translation of the vertices $\{1,3,5,7\}$. By Corollary 6.3 , there are thirty-four 2 -faces, whose arrays contain 3 stars and at most 2 odd inner blocks. These faces are partitioned into ten orbits, seven of which are free orbits and the other three have a stabilizer of order 2, as shown in Figure 4. Note that $X_{1}(q) \equiv 10+7 q+10 q^{2}+7 q^{3}\left(\bmod q^{4}-1\right)$.

\begin{tabular}{|c|c|c|c|c|}
\hline 1234567 & 1234567 & 1234567 & 1234567 & 1234567 \\
\hline$* * *$. & $* * . * \ldots$ & $* * \ldots * \ldots$ & $* * \ldots *$. & $* . * * \ldots$ \\
\hline$. * * . * \ldots$ &.$* * * \ldots$ &.$* * \ldots *$ &.$* * \ldots *$ & $\ldots * * * \ldots$ \\
\hline$. * \ldots * . *$ & $. * . * * \ldots$ & &.$* \ldots * *$ & $\ldots * * * *$ \\
\hline$* * \ldots *$ & **.*..* & &.$* \ldots * *$ & $* \ldots * \ldots *$ \\
\hline$* . * . * \ldots$ & $* \ldots * \ldots$ & $* \ldots * * \ldots$ & $* \ldots * *$. & $* \ldots * *$ \\
\hline$\ldots * * * *$ & $\ldots * . * *$ & $\ldots * * \ldots *$ & $\ldots * * . *$ & $\ldots * \ldots * *$ \\
\hline$* \ldots * \ldots *$ & $\ldots \ldots * * *$ & & $\ldots * * *$ & \\
\hline$* \ldots \ldots *$ & $* \ldots \ldots *$ & & $\ldots * * * *$ & \\
\hline
\end{tabular}

Figure 4: The orbits of the 2 -faces of $\mathrm{CP}(7,5)$ under $\mathbb{Z}_{4}$-action.

\section{Concluding remarks}

In this paper, we prove the CSP for faces of $\mathrm{CP}(n, d)$ for even $d$, along with a natural $q$-analogue $F(n, d, k ; q)$ of the face number $f(n, d, k)$, under an action of the cyclic group $C=\mathbb{Z}_{n}$. For odd $d, C$ is no longer an automorphism group of $\operatorname{CP}(n, d)$ for $n \geqslant d+2$. Inspired by the work of Kaibel and Waßmer [4], we consider automorphism groups $C^{\prime}$ and $C^{\prime \prime}$ of order 2 of $\mathrm{CP}(n, d)$, which are generated by $c^{\prime}=(1, n)(2, n-1) \cdots$ and $c^{\prime \prime}=(1, n)$, respectively. In an attempt on proving the CSP, we derive the number of $(k-1)$-faces that are $C^{\prime}$-invariant (or $C^{\prime \prime}$-invariant). However, so far it lacks a feasible option for the $q$-polynomial, i.e., a relatively natural polynomial $X(q)$ that satisfies the conditions $X(1)=g(n, d, k)$ and $X(-1)=h_{(n, d, k-1)}\left(\right.$ or $\left.X(-1)=z_{(n, d, k-1)}\right)$, as given in Theorem 4.2 
(or Theorem 5.1). It is worth mentioning that the natural $q$-analogue of $g(n, d, k)$ does not work in this situation. For instance, let

$$
X(q)=\sum_{j=1}^{\frac{d+1}{2}} \frac{[k+1]_{q}}{[j]_{q}}\left[\begin{array}{c}
n-j \\
j-1
\end{array}\right]_{q}\left[\begin{array}{c}
j \\
k+1-j
\end{array}\right]_{q} .
$$

It is clear that $X(1)=g(n, d, k)$. In particular, take $k=d$. Note that $h_{(n, d, d-1)}=0$ (i.e., there are no facets of $\mathrm{CP}(n, d)$ that are $C^{\prime}$-invariant). However, one can check that in this case

$X(q)=\left(q^{\frac{d+1}{2}}+1\right)\left[\begin{array}{cl}n-\frac{d+1}{2} \\ \frac{d-1}{2}\end{array}\right]_{q}$, and $X(-1)= \begin{cases}2\left(\begin{array}{c}\left\lfloor\frac{2 n-d-1}{4}\right\rfloor \\ \left\lfloor\frac{d-1}{4}\right\rfloor\end{array}\right) & \text { if } n \text { is odd, } d \equiv 3(\bmod 4) \\ 0 & \text { otherwise. }\end{cases}$

which does not agree with $h_{(n, d, d-1)}$.

For the $C^{\prime \prime}$-case, one may come up with an artificial polynomial $X(q)$ with the properties $X(1)=g(n, d, k)$ and $X(-1)=z_{(n, d, k-1)}$, based on Theorem 5.1, which is defined by

$$
X(q)=g(n, d, k)-(1-q) \cdot f(n-1, d-1, k-1)+(1-q) \cdot g(n-2, d-2, k-2) .
$$

However, the downside is that the polynomial $X(q)$ is always of degree 1 and has no good connection with the group $C^{\prime \prime}$.

Problem 7.1. For odd $d$ and $1 \leqslant k \leqslant d$, let $X$ be the set of $(k-1)$-faces of $\mathrm{CP}(n, d)$. Find a polynomial $X(q)$ with the property $X(1)=g(n, d, k)$ such that the triples $\left(X, X(q), C^{\prime}\right)$ or $\left(X, X(q), C^{\prime \prime}\right)$ exhibit the cyclic sieving phenomenon.

For the special case $n=d+2$ and $d$ is odd, we present two instances of CSP on $\mathrm{CP}(d+2, d)$ with artificial $q$-polynomials $P(d, k ; q)$ and $Q(d, k ; q)$, under the cyclic groups $\mathbb{Z}_{\frac{d+3}{2}}$ and $\mathbb{Z}_{\frac{d+1}{2}}$.

We hope that our proof of the CSP by verifying condition (1) could shed some light on an algebraic proof from the point of view of representation theory.

\section{Acknowledgements}

The authors thank the referee for the careful reading and many helpful suggestions. 


\section{References}

[1] S.-P. Eu, T.-S. Fu, The cyclic sieving phenomenon for faces of generalized cluster complexes, Adv. Appl. Math. 40(3) (2008), 350-376.

[2] D. Gale, Neighborly and cyclic polytopes, In: Proc. Sympos. Pure Math., vol. VII, pp. 225-232, American Mathematical Society, Providence (1963).

[3] B. Grünbaum, Convex polytopes, 2nd edn., Graduate Texts in Mathematics, vol. 221, Springer, New York, 2003.

[4] V. Kaibel, A. Waßmer, Automorphism groups of cyclic polytopes, to appear in: Triangulated Manifolds (Ed. F. Lutz), Springer, New York, 2010.

[5] T.S. Motzkin, Comonotone curves and polyhedra, Bull. Am. Math. Soc. 63 (1957) 35.

[6] B. Sagan, Congruence properties of q-analogs, Adv. Math. 95 (1992) 127-143.

[7] G. C. Shephard, A theorem on cyclic polytopes, Israel J. of Math. 6(4) (1968) 368372.

[8] V. Reiner, D. Stanton, D. White, The cyclic sieving phenomenon, J. Combin. Theory Ser. A 108 (2004) 17-50.

[9] G. M. Ziegler, Lectures on Polytopes, Graduate Texts in Mathematics, vol. 152, Springer, New York, 1995. Revised edition 1998. 